\title{
Second order perturbations in the Randall-Sundrum infinite brane- world model
}

\author{
AUTHOR(S):
}

Kudoh, $\mathrm{H}$; Tanaka, $\mathrm{T}$

\section{CITATION:}

Kudoh, H ...[et al]. Second order perturbations in the Randall-Sundrum infinite brane-world model. PHYSICAL REVIEW D 2001, 64(8): 084022.

\section{ISSUE DATE:}

2001-10-15

URL:

http://hdl.handle.net/2433/49995

RIGHT:

Copyright 2001 American Physical Society 
PHYSICAL REVIEW D, VOLUME 64, 084022

\title{
Second order perturbations in the Randall-Sundrum infinite brane-world model
}

\author{
Hideaki Kudoh* and Takahiro Tanaka ${ }^{\dagger}$ \\ Yukawa Institute for Theoretical Physics, Kyoto University, Kyoto 606-8502, Japan
}

(Received 5 April 2001; published 26 September 2001)

\begin{abstract}
We discuss the nonlinear gravitational interactions in the Randall-Sundrum single brane model. If we naively write down the four-dimensional effective action integrating over the fifth dimension with the aid of the decomposition with respect to the eigenmodes of four-dimensional D'Alembertian, the Kaluza-Klein mode coupling seems to be ill defined. We carefully analyze second order perturbations of the gravitational field induced on the three-brane under the assumption of the static and axial-symmetric five-dimensional metric. It is shown that there remains no pathological feature in the Kaluza-Klein mode coupling after the summation over all different mass modes. Furthermore, the leading Kaluza-Klein corrections are shown to be sufficiently suppressed in comparison with the leading order term that is obtained by the zero mode truncation. We confirm that the four-dimensional Einstein gravity is approximately recovered on the three-brane up to second order perturbations.
\end{abstract}

DOI: 10.1103/PhysRevD.64.084022

PACS number(s): 04.50.+h, 98.80.Cq

\section{INTRODUCTION}

The possibility that our four-dimensional universe is embedded as a brane in a higher dimensional spacetime has been extensively discussed recently as the brane world scenario [1-4]. In particular, Randall and Sundrum introduced attractive models whose background bulk spacetime is fivedimensional anti-de Sitter $\left(\mathrm{AdS}_{5}\right)$ spacetime. These models suggest the possibility of the existence of extra dimensions in a nontrivial form and the possible explanation of the large hierarchy between the Planck scale and the electroweak scale $[3,4]$.

The behavior of gravity in the Randall-Sundrum (RS) models has been investigated by many authors [5-14]. For the RS single brane model, which is a model with the positive tension brane alone, the extension of extra dimensions is infinite. Nevertheless, gravity on the three-brane generated by the matter field, which is confined on the three-brane, approximately coincides with four-dimensional Einstein gravity $[5-8]$.

For the RS two-brane model whose bulk space is bounded by the positive and negative tension branes, four-dimensional Einstein gravity can be recovered on both branes under the approximation of the zero mode truncation [9] when we take the stabilization mechanism of the distance between the two branes into consideration [15-17].

Although a large number of studies have been made on linear perturbations of the metric [18-23], little is known about the nonlinear or nonperturbative feature of gravity [24-30]. There is concern about the RS single brane model. In this model, it seems that the nonlinear gravitational interaction between Kaluza-Klein (KK) modes becomes strong

\footnotetext{
*Email address: kudoh@yukawa.kyoto-u.ac.jp

†Email address: tanaka@yukawa.kyoto-u.ac.jp
}

and it diverges as we move far away from the brane. A more precise argument is that the four-dimensional effective action including interaction terms diverges when we attempt to write it down by integrating over the dependence on the fifth direction by using the decomposition of metric perturbations in terms of the eigenmodes of a four-dimensional D'Alembertian [4]. This fact indicates that we cannot construct a four-dimensional effective action for this model in the usual sense.

The above discussion is based on an analysis using the decomposition of the mass eigenmodes, which is referred to as the mode-by-mode analysis. However, it was demonstrated that the mode-by-mode analysis is insufficient to deal with metric perturbations and it is necessary to take into account the contributions from all the KK modes when we discuss the regularity of linear metric perturbations at a point far from the three-brane [11]. Thus the pathological behavior in the nonlinear interaction can be expected to be an artifact solely due to the mode-by-mode analysis, although it has not been proved yet. Even if the pathological behavior might be fictitious, there is another question as to whether the fourdimensional Einstein gravity is recovered when we proceed to higher order perturbations.

The purpose of this paper is to give a partial answer to the above two questions. To study the nonlinear behavior of the gravity, we investigate second order perturbations in the context of the RS single brane model. To simplify the analysis, we consider the static and axisymmetric configuration, which means that the three-brane metric is spherically symmetric. Following the method developed by Garriga and Tanaka [5], we confirm that there is no pathological feature in the $\mathrm{KK}$ mode coupling if we sum up all the mass eigenmodes, and that the contribution due to the KK mode coupling is sufficiently small compared with the leading order terms, that are obtained by the zero mode truncation. Furthermore, the re- 
sult obtained by the zero mode truncation exactly agrees with the one predicted by four-dimensional Einstein gravity. ${ }^{1}$

This paper is organized as follows. In the next section we first derive the second order perturbation equations in the five-dimensional bulk, and discuss the gauge transformation as well as the boundary condition. In Sec. III, after giving a brief summary of the behavior of the five-dimensional Green function, which is needed to solve the perturbation equations, we review the results for linear perturbations to give their explicit expressions in the notation of the present paper. In Sec. IV, we analyze second order perturbations of the metric induced on the three-brane. First it is shown that the four-dimensional Einstein gravity is recovered in the approximation of the zero mode truncation, and then we prove that the remaining contribution due to the Kaluza-Klein mode coupling can be neglected. Section V is devoted to a summary.

\section{PERTURBATION EQUATIONS IN THE RS MODEL}

The brane-world model proposed by Randall and Sundrum is composed of the five-dimensional AdS space

$$
d s^{2}=g_{a b} d x^{a} d x^{b}=a^{2}(y) \eta_{\mu \nu} d x^{\mu} d x^{\nu}+d y^{2},
$$

with a single positive tension $(\sigma>0)$ three-brane located at $y=0$. Here $a(y) \equiv e^{-|y| / l}$ is the warp factor, and $l$ is the curvature radius of $\mathrm{AdS}_{5}$. We have denoted the fourdimensional Minkowski metric as $\eta_{\mu \nu}$. The cosmological constant on the bulk and the tension of the three-brane are, respectively, related to the curvature radius $l$ as $\Lambda=-6 l^{-2}$ and as $\sigma=3 / 4 \pi l G_{5}$, where $G_{5}$ is the five-dimensional Newton constant. The relation between the five-dimensional and the four-dimensional Newton constants is given by $G_{5}$ $=l G_{4}$. We also use the notation $\kappa_{5} \equiv 8 \pi G_{5}$. Ordinary matter field is supposed to be localized on the brane.

In this paper, we investigate second order perturbations of this model induced by nonrelativistic matter on the brane whose energy momentum tensor is given by the perfect fluid form

\footnotetext{
${ }^{1}$ After we had submitted the previous version of this paper, we had the existence of Ref. [34] pointed out. In this reference, the second order metric perturbation in the region far from the star was investigated using the truncation of the first order metric perturbation at the leading order for the expansion with respect to the distance from the star. Since the Einstein equation was not solved in the whole region of the spacetime in that treatment, there remains an ambiguity in adding some metric perturbations that satisfy the homogeneous linear perturbation equation only in the asymptotic region. Nevertheless, one may be able to prove that the dominant part of this remaining ambiguity can be absorbed by the redefinition of the mass parameter, and actually our results show that this is the case. Hence, the recovery of the 4D Einstein gravity in the region far from the star is to be credited to Ref. [34]. On the other hand, what we show in the present paper is the recovery of the 4D Einstein gravity throughout the whole spacetime including the inside of the star, without assuming a specific radial matter distribution.
}

$$
T_{\mu}^{\nu}=\operatorname{diag}\{-\rho, P, P, P\} .
$$

To simplify the analysis, we restrict our consideration to the static and axisymmetric spacetime whose axis of symmetry lies along the $y$ direction, namely, the three-brane metric is spherically symmetric.

We denote the perturbed metric by $\widetilde{g}_{a b}=g_{a b}+h_{a b}$. At the level of linear perturbations, it is advantageous to use the Randall-Sundrum gauge defined by

$$
\begin{gathered}
h_{y y}=h_{\mu y}=0, \\
h_{\mu ; \nu}^{\nu}=0, \quad h_{\mu}^{\mu}=0,
\end{gathered}
$$

because the linear perturbation equations in this gauge take the simple form [5]

$$
\mathcal{L} h_{\mu \nu} \equiv\left[a^{-2} \square^{(4)}+\partial_{y}^{2}-4 l^{-2}\right] h_{\mu \nu}=0,
$$

and all components are decoupled. However, when we consider second order perturbations, we cannot impose the transverse-traceless condition (2.4) in addition to (2.3). Hence, we need to abandon the condition (2.4) in second order perturbations. As a consequence, the second order perturbation equations are inevitably coupled.

Here, instead of requiring (2.4), we start with the assumption of the diagonal form of the metric

$$
\begin{aligned}
d s^{2}= & a^{2}\left[-e^{\mathcal{A}(r, y)} d t^{2}+e^{\mathcal{B}(r, y)} d r^{2}\right. \\
& \left.+e^{\mathcal{C}(r, y)} r^{2}\left(d \theta^{2}+\sin ^{2} \theta d \varphi^{2}\right)\right]+d y^{2}
\end{aligned}
$$

which is manifestly compatible with the gauge condition (2.3), and does not lose generality under the restriction to the static and axial-symmetric case. Furthermore, we can expect that this assumption is also compatible with the condition (2.4) at the linear order according to the result obtained in Ref. [5]. For the assumed metric form (2.6), the conditions (2.4) at the linear level become, respectively,

$$
\mathcal{A}^{(1)}+\mathcal{B}^{(1)}+2 \mathcal{C}^{(1)}=0,
$$

and

$$
\partial_{r}\left(r^{2} \mathcal{B}^{(1)}\right)-2 r \mathcal{C}^{(1)}=0,
$$

where we have expanded $\mathcal{A}, \mathcal{B}$, and $\mathcal{C}$ to second order as

$$
\mathcal{A}(r, y)=\sum_{J=1,2} \mathcal{A}^{(J)}(r, y) .
$$

Hereafter, we neglect higher order terms without mentioning it.

Before we start to solve the five-dimensional Einstein equation, we would like to mention the boundary condition at $y \rightarrow \infty$. An important point which we must mention here is that we are to find a solution which is regular at $y \rightarrow \infty$. If we allow the violation of regularity at infinity, the dynamics of the RS brane-world model is not uniquely determined. Then, such a model cannot be a candidate for the model that describes our Universe. Hence, we require that the metric con- 
verges to $\mathrm{AdS}_{5}$ asymptotically. To guarantee this asymptotic condition, we simply require that the metric functions $\mathcal{A}, \mathcal{B}$, and $\mathcal{C}$ go to 0 at $y \rightarrow \infty$. With this choice, the residual gauge degrees of freedom are completely fixed. Under this constraint, it is still possible to extend the coordinates throughout the bulk maintaining the diagonal form of the metric. We refer to this gauge choice throughout as the RS gauge because we see later that the first order quantities $\mathcal{A}^{(1)}, \mathcal{B}^{(1)}$, and $\mathcal{C}^{(1)}$ in this gauge satisfy both the conditions (2.7) and (2.8) [although the quantities at the second order do not satisfy the transverse-traceless condition (2.4)].

\section{A. 5D Einstein equations in the bulk}

We consider the five-dimensional Einstein equations and derive the perturbation equations up to second order. Since the trace of metric perturbations vanishes in the RS gauge at linear order, it is convenient to introduce

$$
2 \psi^{(J)} \equiv \mathcal{A}^{(J)}+\mathcal{B}^{(J)}+2 \mathcal{C}^{(J)} .
$$

By using this quantity, the trace of metric perturbations is expressed as

$$
\tilde{g}^{\mu \nu} h_{\mu \nu}=2 \sum_{J=1,2} \psi^{(J)}-\frac{1}{2}\left[\left(\mathcal{A}^{(1)}\right)^{2}+\left(\mathcal{B}^{(1)}\right)^{2}+2\left(\mathcal{C}^{(1)}\right)^{2}\right] .
$$

Hence the traceless condition at first order is simply given by $\psi^{(1)}=0$.

The five-dimensional vacuum Einstein equation with the cosmological term is equivalent to the following set of equations for the Ricci tensor:

$$
\begin{aligned}
R_{y}^{y}+\frac{4}{l^{2}}= & \sum_{J=1,2}\left(\frac{2}{l} \psi_{, y}^{(J)}-\psi_{, y y}^{(J)}\right)-Q_{y y}=0 \\
R_{t}^{t}+\frac{4}{l^{2}}= & \frac{1}{2 l} \sum_{J=1,2}\left(2 \psi_{, y}^{(J)}+4 \mathcal{A}_{, y}^{(J)}-l \mathcal{A}_{, y y}^{(J)}\right. \\
& \left.-l a^{-2} \triangle \mathcal{A}^{(J)}\right)-\frac{1}{2 a^{2}} S=0 \\
R_{r}^{y}= & \frac{1}{2 r} \sum_{J=1,2}\left[\frac{1}{r^{2}}\left(r^{3} \mathcal{B}^{(J)}\right)_{, r y}-2\left(r \psi_{, y}^{(J)}\right)_{, r}+\mathcal{A}_{, y}^{(J)}\right] \\
& +\frac{1}{2} \psi_{, r}^{(1)} \mathcal{B}_{, y}^{(1)}-Q_{r y}=0,
\end{aligned}
$$

where $\triangle \equiv \sum_{i=1}^{3} \partial_{i}^{2}$ and we have defined

$$
S(r, y) \equiv-\frac{1}{r^{2}} \partial_{r}\left(r^{2} \mathcal{A}_{, r}^{(1)} \mathcal{B}^{(1)}\right)+a^{2} \psi_{, y}^{(1)} \mathcal{A}_{, y}^{(1)}+\psi_{, r}^{(1)} \mathcal{A}_{, r}^{(1)},
$$

$Q_{\mu \nu}(r, y) \equiv \frac{1}{4}\left(\mathcal{A}_{, \mu}^{(1)} \mathcal{A}_{, \nu}^{(1)}+\mathcal{B}_{, \mu}^{(1)} \mathcal{B}_{, \nu}^{(1)}+2 \mathcal{C}_{, \mu}^{(1)} \mathcal{C}_{, \nu}^{(1)}\right)$.

Note that no other equations for the remaining components are independent.

First we consider the trace part $\psi^{(J)}$, which can be evaluated by integrating Eq. (2.12). From the requirement of the boundary condition, $\psi^{(J)}$ must go to 0 at $y \rightarrow \infty$. Thus, we obtain

$$
\psi^{(J)}(r, y)=-\epsilon^{(J)} \int_{\infty}^{y} d y^{\prime \prime} e^{2 y^{\prime \prime} / l} \int_{\infty}^{y^{\prime \prime}} d y^{\prime} e^{-2 y^{\prime} / l} Q_{y y} .
$$

where we have introduced a symbol $\epsilon^{(J)}$ that is defined by $\epsilon^{(1)}=0$ and $\epsilon^{(2)}=1$ to represent the first and second order equations in a single expression. As is anticipated above, we can see from Eq. (2.17) that the traceless condition at linear order is actually satisfied, while that at second order can no longer be imposed in general.

Let us now turn to $\mathcal{B}^{(J)}$. Integrating Eq. (2.14), we obtain

$$
\begin{aligned}
\mathcal{B}^{(J)}(r, y)= & -\frac{1}{r^{3}} \int r^{2} \mathcal{A}^{(J)} d r+\frac{2}{r^{3}} \epsilon^{(J)}\left(\int d r r^{2}\left(r \psi^{(2)}\right)_{, r}\right. \\
& \left.+\int_{\infty}^{y} d y \int d r r^{3} Q_{r y}\right) .
\end{aligned}
$$

As before, the integration constant is fixed by the boundary condition at $y \rightarrow \infty$. Now, combined with Eq. (2.7), it is easy to see that Eq. (2.8) holds at linear order. Hence, it is confirmed that our choice of gauge is equivalent to the RS gauge.

So far, we obtained the relations between the metric functions $\mathcal{A}^{(J)}, \mathcal{B}^{(J)}$, and $\mathcal{C}^{(J)}$. Substituting these into Eq. (2.13), we obtain a single equation for $\mathcal{A}^{(J)}$,

$$
\mathcal{L}\left[a^{2} \mathcal{A}^{(J)}\right]=\epsilon^{(J)}\left[2 a^{2} l^{-1} \psi_{, y}-S\right] .
$$

The source terms are absent at linear order, as they are composed of a quadratic in linear order quantities. Since $\psi^{(1)}$ $=0, S$ defined in Eq. (2.15) simplifies as

$$
S(r, y)=\frac{1}{r^{2}} \partial_{r}\left(r^{2} \mathcal{A}_{, r}^{(1)} \mathcal{B}^{(1)}\right) .
$$

\section{B. Boundary condition}

In the previous subsection, we obtained the master equation for the metric functions in the bulk up to second order. To solve this equation, we need to know the boundary condition to be imposed on the three-brane. The boundary condition on the three-brane is specified by Israel's junction condition [31]. However, in the RS gauge defined above, the $y$-constant surface is, by construction, chosen so that the metric functions go to 0 at $y \rightarrow \infty$. After fixing the $y$-constant surface for large $y$, coordinates are extended to the region near the three-brane. Therefore, we can no longer expect that the location of the three-brane coincides with the $y=0$ surface in general [5]. In such coordinates, the junction condition is not so trivial. Thus it will be convenient to introduce other coordinates $\bar{x}^{a}$, in which the location of the three-brane 
stays at $\bar{y}=0$ but the metric form is still kept diagonal. We associate an overbar with the quantities written in these coordinates, say, $\overline{\mathcal{A}}$. We denote this choice of coordinates by the Gaussian normal (GN) gauge. By construction, $\bar{h}_{\mu \nu} \mid \bar{y}_{y}=0$ gives the four-metric induced on the three-brane.

The junction condition on the three-brane is simply written in the GN gauge as ${ }^{2}$

$$
\begin{array}{r}
\widetilde{\sigma}^{\gamma \nu}(x) \partial_{y}\left[a^{-2}(y) \bar{h}_{\mu \nu}(x)\right]=-\kappa_{5}\left(T_{\mu}^{\gamma}-\frac{1}{3} \delta_{\mu}^{\gamma} T_{\lambda}^{\lambda}\right)(\mathbf{x}) \\
(\text { at } y=0+) .
\end{array}
$$

As mentioned earlier, we assume the energy-momentum tensor of the perfect fluid form (2.2). Then, the fourdimensional energy-momentum conservation $T_{\mu ; \nu}^{\nu}=0$ becomes

$$
[\rho(r)+P(r)] \partial_{r} \overline{\mathcal{A}}^{(1)}(r, 0)+2 \partial_{r} P(r)=0,
$$

and hence we find that $P(r)$ is a second order quantity. This equation represents the force balance between pressure and gravity acting on the matter field.

Taking into account that $P(r)$ is second order and expanding $\rho$ as $\rho=\rho^{(1)}+\rho^{(2)}$, the explicit junction conditions for the metric functions become

$$
\begin{aligned}
& \partial_{y} \overline{\mathcal{A}}^{(J)}=\kappa_{5}\left(\frac{2}{3} \rho^{(J)}+\epsilon^{(J)} P\right), \\
& \partial_{y} \overline{\mathcal{B}}^{(J)}=\partial_{y} \overline{\mathcal{C}}^{(J)}=-\frac{\kappa_{5}}{3} \rho^{(J)} \quad(\text { at } y=0+) .
\end{aligned}
$$

The boundary condition obtained above is written in terms of the variables in the GN gauge. To interpret the conditions (2.23) in terms of the variables in the RS gauge, we consider the gauge transformation between these two gauges, which is defined by

$$
\stackrel{\widetilde{g}}{a b}_{a b}(\bar{x}) d \bar{x}^{a} d \bar{x}^{b}=\widetilde{g}_{a b}(x) d x^{a} d x^{b},
$$

with $\bar{x}^{a}=x^{a}+\xi^{a}(x)$. Since $h_{y \mu}$ and $h_{y y}$ vanish in both gauges, the infinitesimal gauge transformation $\xi^{a}=\stackrel{(1)}{\xi^{a}}+\stackrel{(2)}{\xi^{a}}$ between them is restricted to the following form:

$$
\stackrel{(J)}{\xi^{y}}(r, y)=\stackrel{(J)}{\hat{\xi}^{y}}(r)-\epsilon^{(J)}\left[\frac{l}{4 a^{2}}\left(\hat{\xi}_{, r}^{y}\right)^{2}\right],
$$

\footnotetext{
${ }^{2}$ One may think that this equation should have been expressed as

$$
\widetilde{\bar{g}}^{\gamma \nu}(\bar{x}) \partial_{y}\left[a^{-2}(\bar{y}) \bar{h}_{\mu \nu}(\bar{x})\right]=-\kappa_{5}\left(T_{\mu}^{\gamma}-\frac{1}{3} \delta_{\mu}^{\gamma} T_{\lambda}^{\lambda}\right)(\overline{\mathbf{x}}) \quad(\text { at } \bar{y}=0+),
$$
}

because this is a relation for the quantities in the GN gauge. However, $\left.f(x)\right|_{y=0}=0$ is obviously equivalent to $\left.f(\bar{x})\right|_{\bar{y}=0}=0$ for any function $f$. To stress this point, we always use the coordinates without overbar hereafter. The only exception is Eq. (2.24).

$$
\begin{aligned}
\xi^{r}(r, y)= & \stackrel{(J)}{\hat{\xi}^{r}}(r)-\frac{l}{2 a^{2}} \hat{\xi}_{, r}^{(J)}+\epsilon^{(J)} \hat{\xi}_{, r}^{y}\left[\frac{l}{2 a^{2}}\left(\begin{array}{l}
(1) \\
\hat{\xi}_{, r}^{r}-\frac{2}{l} \hat{\xi}^{y}
\end{array}\right)\right. \\
& \left.+\int_{0}^{y} \frac{d y^{\prime}}{a^{2}} \overline{\mathcal{B}}^{(1)}\left(r, y^{\prime}\right)\right] .
\end{aligned}
$$

Functions $\stackrel{(J)}{\hat{\xi}^{y}}(r)$ and $\stackrel{(J)}{\hat{\xi}^{r}}(r)$ are integration constants which appear as a result of $y$ integration. We discuss how these functions are determined later.

We denote the difference between the metric in the RS gauge and that in the GN gauge as

$$
\delta \mathcal{A}^{(J)}(r, y) \equiv \mathcal{A}^{(J)}(r, y)-\overline{\mathcal{A}}^{(J)}(r, y)
$$

The remaining metric functions $\delta \mathcal{B}^{(J)}$ and $\delta \mathcal{C}^{(J)}$ are defined in the same way. Then, the gauge transformations are given by

$$
\begin{aligned}
& \delta \mathcal{A}^{(J)}=-\frac{2^{(J)}}{l} \xi^{y}+\epsilon^{(J)}\left[\overline{\mathcal{A}}_{, y}^{(1)} \xi^{(1)}+\overline{\mathcal{A}}_{, r}^{(1)} \xi^{(1)}\right] \\
& \delta \mathcal{B}^{(J)}=-\frac{2^{(J)}}{T} \xi^{y}+2{\stackrel{(J)}{\xi_{, r}^{r}}}^{(J} \epsilon^{(J)}\left[\overline{\mathcal{B}}_{, y}^{(1)}{ }^{(1)} \xi^{y}+\overline{\mathcal{B}}_{, r}^{(1)} \stackrel{(1)}{ }^{r}-\left({ }^{r}{ }_{, r}^{r}\right)^{2}\right. \\
& \text { (1) } \\
& \left.+e^{2 y / l}\left(\xi_{, r}^{y}\right)^{2}\right] \\
& \delta \mathcal{C}^{(J)}=-\frac{2^{(J)}}{l} \xi^{y}+\frac{2^{(J)}}{r} \xi^{r}+\epsilon^{(J)}\left[\overline{\mathcal{C}}_{, y}^{(1)} \xi^{(1)}+\overline{\mathcal{C}}_{, r}^{(1)} \xi^{(1)}-\left(\frac{1}{r} \xi^{r}\right)^{2}\right] .
\end{aligned}
$$

Now we are ready to derive the equation that determines $\hat{\xi}^{y}(r)$. We evaluate the identity $\psi_{, y}=\bar{\psi}_{, y}+\delta \psi_{, y}$ at $y=0$. The expression for the left hand side is obtained by means of Eq. (2.17), while the right hand side is evaluated by substituting Eqs. (2.23), (2.25), (2.26), and (2.28). After tedious but straightforward computation, we obtain

$$
\triangle \hat{\xi}^{y}=\frac{\kappa_{5}}{6} T^{(J)}+\epsilon^{(J)}\left[\Xi(r)-\left.\psi_{, y}\right|_{y=0}\right]
$$

with

$$
\begin{aligned}
\Xi(r) \equiv & \Delta f t\left[\frac{1}{T}\left(\hat{\xi}^{y}\right)^{2}+\int_{\infty}^{r} \hat{\xi}_{, r}^{(1)} \mathcal{B}^{(1)}\left(r^{\prime}, 0\right) d r^{\prime}\right]-\frac{3}{T}\left(\hat{\xi}_{, r}^{y}\right)^{2} \\
& +\hat{\xi}^{r} \partial_{r} \triangle \hat{\xi}^{y}+\frac{l}{2}\left(\hat{\xi}_{, r r}^{y}\right)^{2}+\frac{l}{r^{2}}\left(\hat{\xi}_{, r}^{y}\right)^{2}
\end{aligned}
$$

The gauge freedom for the radial coordinate in the GN gauge has not been fixed. Although the simplest choice might be to take $\xi^{r}$ to vanish on the brane, for later convenience we impose the isotropic gauge condition on the threebrane, $\overline{\mathcal{B}}=\overline{\mathcal{C}}$ at $\bar{y}=0$. Rewriting this condition by using $\mathcal{B}$ and $\mathcal{C}$ with the substitution of Eq. (2.28), we obtain 


$$
\stackrel{(1)}{\xi^{r}}=-\frac{r}{4} \mathcal{B}^{(1)}
$$

Here we show the result only for the first order, because we (2)

do not need the explicit expression for $\xi^{r}$ in the following discussion.

Once we obtain an explicit expression for $\hat{\xi}^{y}$, it is easy to derive the boundary condition for the metric functions in the RS gauge from the junction condition in the GN gauge (2.23). In particular, the boundary condition required to solve the master equation (2.19) is deduced by substituting the relations obtained above into the right hand side of the equation $\mathcal{A}_{, y}=\overline{\mathcal{A}}_{, y}+\delta \mathcal{A}_{, y}$. Imposing this boundary condition is equivalent to adding a $\delta$-function source localized on the three-brane. The five-dimensional master equation (2.19) including the boundary condition becomes

$$
\begin{aligned}
{[\mathcal{L}+} & \left.4 l^{-1} \delta(y)\right]\left(a^{2} \mathcal{A}^{(J)}\right) \\
& =2 \kappa_{5} \Sigma^{(J)}(r) \delta(y)+\epsilon^{(J)}\left[2 a^{2} l^{-1} \psi_{, y}-S\right],
\end{aligned}
$$

where

$$
\begin{aligned}
\Sigma^{(J)}(r) & \left.\equiv \kappa_{5}^{-1} \partial_{y} \mathcal{A}^{(J)}\right|_{y=0} \\
& =\frac{2}{3} \rho^{(J)}+\epsilon^{(J)}\left[P+\left.\kappa_{5}^{-1} \partial_{y}\left(\delta \mathcal{A}^{(2)}\right)\right|_{y=0}\right]
\end{aligned}
$$

By using the Green function which satisfies

$$
\left[\mathcal{L}+4 l^{-1} \delta(y)\right] G\left(\mathbf{x}, y ; \mathbf{x}^{\prime}, y^{\prime}\right)=\delta\left(y-y^{\prime}\right) \delta^{3}\left(\mathbf{x}-\mathbf{x}^{\prime}\right)
$$

the formal solution for the master equation (2.32) is given by

$$
\begin{aligned}
a^{2} \mathcal{A}^{(J)}(r, y)= & 2 \kappa_{5} \int d x^{\prime 3} G\left(\mathbf{x}, y ; \mathbf{x}^{\prime}, 0\right) \Sigma^{(J)} \\
& -2 \epsilon^{(J)} \int d^{3} x^{\prime} \int_{0}^{\infty} d y^{\prime} G\left(\mathbf{x}, y ; \mathbf{x}^{\prime}, y^{\prime}\right) \\
& \times\left[S\left(r^{\prime}, y^{\prime}\right)+\frac{2}{l} \int_{\infty}^{y^{\prime}} a^{2}\left(y^{\prime \prime}\right) Q_{y y}\left(r^{\prime}, y^{\prime \prime}\right) d y^{\prime \prime}\right] .
\end{aligned}
$$

The factor 2 in the second term of Eq. (2.35) comes from $Z_{2}$ symmetry. In Sec. III we discuss some basic aspects of the Green function, and solve the above equation at linear order. In Sec. IV we extend our analysis to the second order.

\section{GREEN FUNCTION AND FIRST ORDER PERTURBATIONS}

\section{A. Green function}

We need the Green function to evaluate explicitly the formal solution (2.35). In the static case the Green function is

$$
\begin{aligned}
G\left(\mathbf{x}, y ; \mathbf{x}^{\prime}, y^{\prime}\right)= & -\frac{1}{4 \pi l R}\left(a^{2}(y) a^{2}\left(y^{\prime}\right)\right. \\
& \left.+l \int_{0}^{\infty} u_{m}(y) u_{m}\left(y^{\prime}\right) e^{-m R} d m\right),
\end{aligned}
$$

where $u_{m}(y)$ is the mode function, and we have introduced the notation

$$
R \equiv\left|\mathbf{x}-\mathbf{x}^{\prime}\right|
$$

The explicit form of the mode function is given in terms of Bessel functions as

$u_{m}(y)=N_{m}\left[J_{1}(m l) Y_{2}(m l / a)-Y_{1}(m l) J_{2}(m l / a)\right]$,

with $N_{m}=\sqrt{m l} / \sqrt{2\left[J_{1}(m l)^{2}+Y_{1}(m l)^{2}\right]}$. It is orthonormalized as

$$
2 \int_{0}^{\infty} \frac{d y}{a^{2}} u_{m} u_{m^{\prime}}=\delta\left(m-m^{\prime}\right) .
$$

In particular, setting $m^{\prime}=0$, we have

$$
\int_{0}^{\infty} d y u_{m}=0 \quad(m \neq 0) .
$$

The first term on the right hand side of Eq. (3.1) is the contribution from the zero mode whose four-dimensional mass eigenvalue is zero $(m=0)$. We denote this part of Green function by $G_{0}$. The second term corresponds to the propagator due to the Kaluza-Klein states which have nonzero mass eigenvalues $(m>0)$. This term is denoted by $G_{K}$. Thus, $G=G_{0}+G_{K}$.

For large separation $R \gg l$, the existence of the factor $e^{-m R}$ in the second term in Eq. (3.1), i.e., $G_{K}$, implies that the integral over $m$ is dominated by the contribution from small $m$. When the source is on the three-brane $\left(y^{\prime}=0\right)$, we can approximately evaluate $G_{K}$ by expanding Bessel functions taking $m l$ as small, but here the Bessel function with the argument $m l / a$ is to be kept unexpanded because $a$ can be exponentially small. Then, the integration over $m$ can be performed for the leading power of $m$ explicitly. The result is given by [5]

$$
G\left(\mathbf{x}, y ; \mathbf{x}^{\prime}, 0\right) \approx-\frac{a^{3}}{8 \pi l} \frac{\left(2 a^{2} R^{2}+3 l^{2}\right)}{\left(a^{2} R^{2}+l^{2}\right)^{3 / 2}} .
$$

For a small separation $R \ll l$, the Green function is dominated by the contribution from modes with large $m$. In this limit, the Green function behaves as the ordinary five-dimensional one $\approx-1 / 4 \pi^{2}\left[R^{2}+l^{2}\left(1-a^{-1}\right)^{2}\right]$.

When we discuss second order perturbations later, we use the following inequalities:

$$
0 \leqslant-G\left(\mathbf{x}, y ; \mathbf{x}^{\prime}, 0\right) \leqslant \frac{a^{2}(y)}{4 \pi l R^{2}}(R+l)
$$




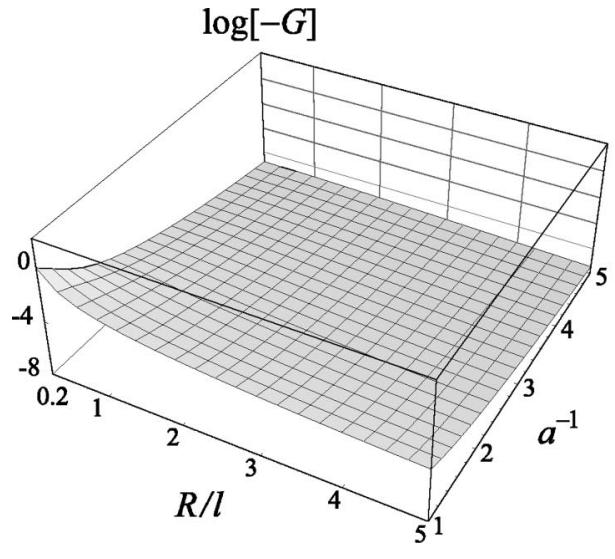

This inequality is suggested by the asymptotic form of the Green function, and it is confirmed by numerical calculations as shown in Fig. 1.

\section{B. Linear perturbations}

We start with linear perturbations. From Eq. (2.35), $\mathcal{A}^{(1)}$ is given by

$$
a^{2} \mathcal{A}^{(1)}=\frac{4 \kappa_{5}}{3} \int d^{3} x^{\prime} G\left(\mathbf{x}, y ; \mathbf{x}^{\prime}, 0\right) \rho^{(1)}\left(r^{\prime}\right) .
$$

Suppose that $r_{*}$ is the radius of the star. When we consider the metric at a point outside the star $r-r_{*} \gg l$ or far from the three-brane, we can safely replace $G$ by the approximation (3.5) with the relative error of $O\left(l^{2} / r_{*}^{2}\right){ }^{3}$ Furthermore, at a field point far from the star, the matter distribution can be replaced with a point source. Then, we obtain $\mathcal{A}^{(1)}(r, y) \sim$ $-4 G_{4} M a\left(2 a^{2} r^{2}+3 l^{2}\right) / 3\left(a^{2} r^{2}+l^{2}\right)^{3 / 2}$. By using Eq. (2.18), we find $\mathcal{B}^{(1)}(r, y) \sim 4 G_{4} M a / 3 \sqrt{a^{2} r^{2}+l^{2}}$.

On the other hand, if we are interested in the metric induced on the brane, we can also set $y=0$. Even if we consider the interior of the star, the inequality (3.6) implies that the contribution to $\mathcal{A}^{(1)}$ from $G_{K}$ is, at most, of $O\left(\left(\kappa_{4} M_{*} / r_{*}\right)\left(l^{2} / r_{*}^{2}\right) \log \left(l / r_{*}\right)\right)$, where $M_{*} \equiv 4 \pi \int r^{2} \rho(r) d r$. Hence, it is small by a factor of $O\left(\left(l^{2} / r_{*}^{2}\right) \log \left(l / r_{*}\right)\right)$ compared with the leading term. Then, neglecting the collections of this order or higher, we obtain

$$
\begin{aligned}
& \mathcal{A}^{(1)}(r, 0) \approx \frac{8}{3} \phi, \\
& \mathcal{B}^{(1)}(r, 0) \approx-\frac{8}{3 r} \partial_{r} \triangle^{-1} \phi,
\end{aligned}
$$

where $\triangle^{-1}$ is the inverse of the Laplacian operator, and we have introduced the Newtonian potential

$$
\phi \equiv 4 \pi G_{4} \triangle^{-1} \rho .
$$

\footnotetext{
${ }^{3}$ Here $r_{*}$ in the denominator is just inserted to adjust the dimensionality. It can be $r$ instead of $r_{*}$.
}

These are transformed to the isotropic GN gauge by using Eq. (2.28) with

$$
\stackrel{(1)}{\hat{\xi}^{y}}=-\frac{l \phi}{3}, \quad \stackrel{(1)}{\xi^{r}} \approx \frac{2}{3} \partial_{r} \triangle^{-1} \phi,
$$

which are derived from Eqs. (2.29) and (2.31), respectively. Finally, the resulting metric functions turn out to be

$$
-\overline{\mathcal{A}}^{(1)}(r, 0) \approx \overline{\mathcal{B}}^{(1)}(r, 0)=\overline{\mathcal{C}}^{(1)}(r, 0) \approx-2 \phi(r),
$$

which agree with the result for the four-dimensional Einstein gravity.

\section{SECOND ORDER PERTURBATIONS}

For later convenience, we quote the result obtained in the preceding section as

$\mathcal{A}^{(2)}(r, 0) \equiv A_{\Sigma}+A_{S}+A_{Q}-\frac{2}{l} \triangle^{-1} \int_{0}^{\infty} d y^{\prime} a^{2} Q_{y y}$,

where

$A_{\Sigma}=2 \kappa_{5} \int d x^{\prime 3} G\left(\mathbf{x}, 0 ; \mathbf{x}^{\prime}, 0\right) \Sigma^{(2)}$

$A_{S}=2 \int d^{3} x^{\prime} \int_{0}^{\infty} d y^{\prime} G\left(\mathbf{x}, 0 ; \mathbf{x}^{\prime}, y^{\prime}\right) \frac{1}{r^{\prime 2}} \partial_{r^{\prime}}\left(r^{\prime 2} \mathcal{A}_{, r^{\prime}}^{(1)}\left(r^{\prime}, y^{\prime}\right)\right.$

$\left.\times \mathcal{B}^{(1)}\left(r^{\prime}, y^{\prime}\right)\right)$,

$A_{Q}=\frac{4}{l} \int d^{3} x^{\prime} \int_{0}^{\infty} d y^{\prime}\left[\int_{\infty}^{y^{\prime}} G\left(\mathbf{x}, 0 ; \mathbf{x}^{\prime}, y^{\prime \prime}\right) d y^{\prime \prime}\right] a^{2}\left(y^{\prime}\right)$

$\times Q_{y y}\left(r^{\prime}, y^{\prime}\right)$.

The source term $\Sigma^{(2)}$ for $A_{\Sigma}$ is given in Eq. (2.33). The third term in Eq. (2.35) is separated into two pieces, $A_{Q}$ and the last term in Eq. (4.1), by performing integration by parts. To obtain the expression of this last term, we have also used $\int d^{3} x^{\prime} \int_{0}^{\infty} G d y^{\prime}=\int d^{3} x^{\prime} \int_{0}^{\infty} G_{0} d y^{\prime}=(1 / 2) \triangle^{-1}$. Here the first equality follows from Eq. (3.4). 
Since we are interested in the metric induced on the threebrane, we also write down the expression for $\overline{\mathcal{A}}^{(2)}$ at $y=0$. Combining Eq. (2.25), (2.28), (2.29), and (4.1), we obtain

$$
\begin{aligned}
\overline{\mathcal{A}}^{(2)}(r, 0)= & A_{\Sigma}+A_{S}+A_{Q}+\frac{\kappa_{4}}{3} \Delta^{-1} T^{(2)} \\
& +\left[\frac{2}{T} \triangle^{-1} \Xi-\frac{1}{2}\left(\xi_{, r}^{(1)}\right)^{2}-\overline{\mathcal{A}}_{, y}^{(1)} \xi^{y}-\overline{\mathcal{A}}_{, r}^{(1)} \xi^{r}\right]_{y=0} .
\end{aligned}
$$

Here we note that the last term in Eq. (4.1) is canceled with a term which arises from the gauge transformation.

The terms in the square brackets are evaluated just by substituting the estimate at first order, and the first term $A_{\Sigma}$ has structure similar to first order perturbations, i.e., the source term is localized on the brane. Thus, the evaluation of these terms is straightforward. What needs detailed investigation is the evaluation of $A_{S}$ and $A_{Q}$. Since the source terms of $A_{S}$ and $A_{Q}$, which are quadratic in the linear perturbation quantities, distribute through the five-dimensional bulk, it is necessary to evaluate a convolution of three fivedimensional Green functions.

Deferring the estimate of this convolution until Sec. IV B, let us turn to the spatial components of the second order metric perturbations. Although each spatial component de(2)

pends on the choice of $\xi^{r}$, the gauge invariant combination

$$
\begin{aligned}
\left\{\overline{\mathcal{B}}^{(2)}-\right. & \left.\partial_{r}\left(r \overline{\mathcal{C}}^{(2)}\right)\right\}_{y=0} \\
= & \left\{\mathcal{B}^{(2)}-\partial_{r}\left(r \mathcal{C}^{(2)}\right)-\delta \mathcal{B}^{(2)}+\partial_{r}\left(r \delta \mathcal{C}^{(2)}\right)\right\}_{y=0} \\
= & \left\{\frac{r}{2} \overline{\mathcal{A}}_{, r}^{(2)}+\frac{r}{2} \partial_{r} \delta \mathcal{A}^{(2)}-\delta \mathcal{B}^{(2)}+\partial_{r}\left(r \delta \mathcal{C}^{(2)}\right)\right\}_{y=0} \\
& -r \int_{0}^{\infty} d y Q_{y r}
\end{aligned}
$$

(2)

does not contain $\xi^{r}$. Here we have used Eqs. (2.10) and (2.18) with $y=0$ to eliminate $\mathcal{B}$ and $\mathcal{C}$. Taking the isotropic gauge $\overline{\mathcal{B}}^{(2)}=\overline{\mathcal{C}}^{(2)}$, the left hand side becomes $-r \overline{\mathcal{B}}_{, r}^{(J)}$. Integrating this equation with respect to $r$, we obtain the expression for $\overline{\mathcal{B}}^{(2)}\left(=\overline{\mathcal{C}}^{(2)}\right)$. Here we note that the terms in the (2)

square brackets in Eq. (4.4) contain $\xi^{r}$, from which a term with $y$ integration,

$$
-\frac{3}{l} \int_{0}^{\infty} d y a^{2} Q_{y y},
$$

arises. Combined with the last term with $y$ integration in Eq. (4.4), this term is reduced to the expression that does not contain $y$ integration. The detail of calculation is explained in Appendix A. Here we just quote the final result:

$$
\begin{aligned}
\overline{\mathcal{B}}^{(2)}(r, 0)= & -\frac{1}{2} \overline{\mathcal{A}}^{(2)}+\triangle^{-1}\left(\frac{\kappa_{4}}{2} T^{(2)}+\frac{3}{l} \Xi+\frac{1}{2} Q_{y y}\right. \\
& \left.-\frac{3}{16}\left(\mathcal{A}_{, r}^{(1)}\right)^{2}\right)+B_{\Sigma} \quad(\text { at } \quad y=0),
\end{aligned}
$$

where

$$
\begin{aligned}
B_{\Sigma} \equiv & -\frac{3}{4}\left(\hat{\xi}_{, r}^{y}\right)^{2}+\int d r \frac{1}{r}\left(\hat{\xi}_{, r}^{y}\right)^{2}+\frac{l \kappa_{4}}{4} \Delta^{-1} \\
& \times\left[\left(3 \mathcal{A}_{, y}^{(1)}+\mathcal{B}_{, y}^{(1)}\right) \Sigma^{(1)}\right. \\
& \left.-\frac{1}{r^{3}}\left(3 \mathcal{B}_{, y}^{(1)}+\mathcal{A}_{, y}^{(1)}\right) \int r^{2} \Sigma^{(1)} d r\right]_{y=0} .
\end{aligned}
$$

We find that the expression is reduced to a closed form written solely in terms of the quantities on the three-brane except for $\overline{\mathcal{A}}^{(2)}(r, 0)$.

\section{A. Recovery of the four-dimensional Einstein gravity}

In this subsection, we evaluate the metric induced on the three-brane at the leading order in $l / r_{*}$, and show that the result completely agrees with the one predicted by the fourdimensional Einstein gravity.

As for $A_{\Sigma}$, since the source term is localized on the threebrane, we can approximate it as

$$
A_{\Sigma} \approx 2 \kappa_{4} \triangle^{-1} \Sigma^{(2)}
$$

which is justified for the same reason explained in evaluating the induced metric at the linear order.

As mentioned earlier, the point that needs careful analysis is the computation of $A_{S}$ and $A_{Q}$. To evaluate these terms we need to evaluate a convolution of the five-dimensional Green functions. Since $G$ is composed of $G_{0}$ and $G_{K}$, the contribution from these terms is decomposed into several pieces depending on which combination of three propagators is used. For example, there is a mode coupling in which a zero mode propagator $G_{0}$ propagates the second order source produced by a product of KK mode contributions. We denote this mode coupling as

$$
[K, K ; 0] \text {, }
$$

and similar labels are assigned for the other mode couplings, too.

Here in the present subsection, we simply neglect the pieces containing the $\mathrm{KK}$ propagator $G_{K}$. That is, we just take into account $A_{S[0,0 ; 0]}$ and $A_{Q[0,0 ; 0]}$. The justification of this approximation is given in the next subsections, where we prove that the neglected pieces in $A_{S}$ and $A_{Q}$ containing $G_{K}$ are actually suppressed by a factor of $O\left(\left(l^{2} / r_{*}^{2}\right) \log \left(r_{*} / l\right)\right)$.

As long as only the contributions from the zero mode are concerned, the first order metric functions are all constant in $y$. Since the source term of $A_{Q}$ contains differentiation of the 
first order metric functions with respect to $y$, we find that $A_{Q[0,0 ; 0]} \approx 0$. As for $A_{S[0,0 ; 0]}$, after the $y$ integration, we find that it is reduced to

$$
A_{S} \approx \triangle^{-1} \frac{1}{r^{2}} \partial_{r}\left(r^{2} \mathcal{A}_{, r}^{(1)} \mathcal{B}^{(1)}\right) .
$$

Now that the evaluation of $\overline{\mathcal{A}}^{(2)}(r, 0)$ is straightforward. Substituting the evaluations of the first order quantities presented in Sec. III B, we finally obtain

$$
\overline{\mathcal{A}}^{(2)}(r, 0) \approx \kappa_{4} \triangle^{-1}\left(\rho^{(2)}-2 \phi \rho^{(1)}+3 P\right),
$$

and

$$
\overline{\mathcal{B}}^{(2)}(r, 0)=\overline{\mathcal{C}}^{(2)}(r, 0) \approx-\Delta^{-1}\left[\kappa_{4}\left(\rho^{(2)}-2 \phi \rho^{(1)}\right)+\left(\phi_{, r}\right)^{2}\right] .
$$

It must be noted that $B_{\Sigma}$ in Eq. (4.5) gives only higher order correction of $O\left(l^{2} \kappa_{4}^{2}\right)$ as is easily shown by using Eq. (2.23), (2.28), (2.29), and (2.31). These results agree with those for the four-dimensional Einstein gravity, whose brief derivation is given in Appendix B.

\section{B. Suppression of the KK mode propagation}

In the preceding subsection the terms in $A_{S}$ and $A_{Q}$ containing a KK mode propagator were neglected. Here we show that the contribution from these terms is in fact negligible. We begin with discussing rather general things. As for $A_{S}$, the terms $A_{S[0,0 ; K]}, A_{S[0, K ; 0]}$, and $A_{S[K, 0 ; 0]}$ vanish because of the orthogonality (3.4). Hence the terms to be investigated are (1) $A_{S[K, K ; 0]}, A_{S[0, K ; K]}, A_{S[K, 0 ; K]}$, and $A_{S[K, K ; K]}$. As for $A_{Q}$, the situation is a little simpler. Recall the fact mentioned above that the zero mode contribution in the first order metric functions is $y$ independent. Thus we can say that $A_{Q[0, * ; *]}$ and $A_{Q[*, 0 ; *]}$ vanish. Therefore, all the terms that we need to consider for $A_{Q}$ are (2) $A_{Q[K, K ; 0]}$ and $A_{Q[K, K ; K]}$.

\section{1. $A_{S[K, K ; 0]}, A_{S[K, 0 ; K]}$, and $A_{S[0, K ; K]}$}

The source term of $A_{S}$ is rewritten as

$$
\begin{aligned}
\frac{1}{r^{2}} \partial_{r}\left(r^{2} \mathcal{A}_{, r}^{(1)} \mathcal{B}^{(1)}\right) \\
=\frac{1}{r_{*}^{2}}\left\{\left\{\left(r_{*}^{2} \partial_{r}^{2}-\frac{r_{*}^{2}}{r} \partial_{r}\right) \mathcal{A}^{(1)}\right\}\left(-\frac{1}{r^{3}}{ }^{r} d r r^{2} \mathcal{A}^{(1)}\right)\right. \\
\left.-\left(\frac{r_{*}^{2}}{r} \partial_{r} \mathcal{A}^{(1)}\right) \mathcal{A}^{(1)}\right] .
\end{aligned}
$$

Each source term has the form of

$$
\left(\mathcal{O}_{r}^{[1]} \mathcal{A}^{(1)}\right)\left(\mathcal{O}_{r}^{[2]} \mathcal{A}^{(1)}\right)
$$

with $\mathcal{O}_{r}^{[1,2]}=1,\left(r_{*}^{2} / r\right) \partial_{r}, r_{*}^{2} \partial_{r}^{2}$, or $r^{-3} \int_{0}^{r} d r r^{2}$. By using the orthonormal condition (3.3), we obtain the formula

$$
\begin{gathered}
\int_{0}^{\infty} d y a^{-2} G_{K}\left(\mathbf{x}_{1}, y ; \mathbf{x}_{2}, 0\right) G_{K}\left(\mathbf{x}_{3}, y ; \mathbf{x}_{4}, 0\right) \\
=\frac{1}{2(4 \pi)^{2} R_{12} R_{34}} \mu\left(R_{12}+R_{34}\right),
\end{gathered}
$$

where $R_{A B} \equiv\left|\mathbf{x}_{A}-\mathbf{x}_{B}\right|$ and

$$
\mu(s) \equiv \int_{0}^{\infty} d m u_{m}(0)^{2} e^{-m s} .
$$

The function $\mu(s)$ is bounded by

$$
0 \leqslant \mu(s)<\frac{l}{2 s(s+l)} .
$$

We begin with $A_{S[K, K ; 0]}+A_{S[K, K ; K]}$. We can derive an upper bound for the absolute value of each term as

$$
\begin{aligned}
\mid \frac{2}{r_{*}^{2}} \int & d^{3} x^{\prime} \int_{0}^{\infty} d y^{\prime} G\left(\mathbf{x}, 0 ; \mathbf{x}^{\prime}, y^{\prime}\right) \\
\times & {\left[\mathcal{O}_{r_{1}}^{[1]} \mathcal{A}_{K}^{(1)}\left(r_{1}, y^{\prime}\right) \mathcal{O}_{r_{3}}^{[2]} \mathcal{A}_{K}^{(1)}\left(r_{3}, y^{\prime}\right)\right]_{r_{1}=r_{3}=r^{\prime}} \mid } \\
< & \frac{1}{8 \pi l r_{*}^{2}} \int d^{3} x^{\prime} \int_{0}^{\infty} d y^{\prime} \frac{a^{2}\left(y^{\prime}\right)}{R^{2}}(R+l) \\
& \times\left[\left\{\mathcal{O}_{r_{1}}^{[1]} \mathcal{A}_{K}^{(1)}\left(r_{1}, y^{\prime}\right)+\mathcal{O}_{r_{1}}^{[2]} \mathcal{A}_{K}^{(1)}\left(r_{1}, y^{\prime}\right)\right\}^{2}\right. \\
& \left.+\left\{\mathcal{O}_{r_{1}}^{[1]} \mathcal{A}_{K}^{(1)}\left(r_{1}, y^{\prime}\right)-\mathcal{O}_{r_{1}}^{[2]} \mathcal{A}_{K}^{(1)}\left(r_{1}, y^{\prime}\right)\right\}^{2}\right]_{r_{1}=r^{\prime}},
\end{aligned}
$$

where $\mathcal{A}_{K}^{(1)}$ is a part of $\mathcal{A}^{(1)}$ propagated by the KK mode propagator, and we have used Eq. (3.6). Below, we show that each term on the right hand side of the above inequality is at most $O\left(l^{2} \log \left(r_{*} / l\right)\right)$. We express the appropriate term as

$$
\frac{1}{r_{*}^{2}} \int d^{3} x^{\prime} \frac{R+l}{R^{2}}\left[\mathcal{O}_{r_{1}}^{[1]} \mathcal{O}_{r_{3}}^{[2]} v\left(r_{1}, r_{3}\right)\right]_{r_{1}=r_{3}=r^{\prime}},
$$

with

$$
\begin{aligned}
v\left(r_{1}, r_{3}\right) \equiv & l^{-1} \int_{0}^{\infty} d y a^{2} \mathcal{A}_{K}^{(1)}\left(r_{1}\right) \mathcal{A}_{K}^{(1)}\left(r_{2}\right) \\
= & \frac{\kappa_{4}^{2} l}{2 r_{1} r_{3}} \int_{0}^{r_{*}} d r_{2} r_{2} \Sigma^{(1)}\left(r_{2}\right) \int_{0}^{r_{*}} d r_{4} r_{4} \Sigma^{(1)}\left(r_{4}\right) \\
& \times \int_{\left|r_{1}-r_{2}\right|}^{r_{1}+r_{2}} d R_{12} \int_{\left|r_{3}-r_{4}\right|}^{r_{3}+r_{4}} d R_{34} \mu\left(R_{12}+R_{34}\right),
\end{aligned}
$$

where $v\left(r_{1}, r_{3}\right)$ has been rewritten by using Eq. (4.12). Introducing $U(s) \equiv-\int_{0}^{s} d s^{\prime} \int_{\infty}^{s^{\prime}} d s^{\prime \prime} \mu\left(s^{\prime \prime}\right)$, 


$$
\begin{aligned}
v\left(r_{1}, r_{3}\right)= & -\frac{\kappa_{4}^{2} l}{2 r_{1} r_{3}} \int_{0}^{r_{*}} d r_{2} r_{2} \Sigma^{(1)}\left(r_{2}\right) \int_{0}^{r_{*}} d r_{4} r_{4} \Sigma^{(1)}\left(r_{4}\right) \\
& \times\left(U\left(r_{1}+r_{2}+r_{3}+r_{4}\right)\right. \\
& -U\left(\left|r_{1}-r_{2}\right|+r_{3}+r_{4}\right)-U\left(r_{1}+r_{2}+\left|r_{3}-r_{4}\right|\right) \\
& \left.+U\left(\left|r_{1}-r_{2}\right|+\left|r_{3}-r_{4}\right|\right)\right) .
\end{aligned}
$$

By construction, $U(0)=0$. The inequality (4.14) indicates that

$$
0 \leqslant U(s) \leqslant \frac{1}{2}\left[s \log \left(1+\frac{l}{s}\right)+l \log \left(1+\frac{s}{l}\right)\right]
$$

As long as $s \lesssim r_{*}$, we find that $U(s)$ is at most $O\left(l \log \left(r_{*} / l\right)\right)$.

Let us consider the action of differentiation once or twice with respect to $r_{1}$ on $U$. Since $r_{1}$ appears in the arguments of $U$ only in the combination of $r_{1}+r_{2}$ or $\left|r_{1}-r_{2}\right|$, the differentiation with respect to $r_{1}$ can be replaced with that with respect to $r_{2}$. Then, integrating by parts, finally the differentiation can be moved so that it acts on the source term $r_{2} \Sigma^{(1)}\left(r_{2}\right)$. As long as the first or second derivative is concerned, differentiation of $U$ does not appear as the boundary term. The same thing is true for the pair of $r_{3}$ and $r_{4}$. Now, using the bound for $U$ obtained above, we can conclude that $\mathcal{O}_{r_{1}}^{[1]} \mathcal{O}_{r_{3}}^{[2]} v\left(r_{1}, r_{3}\right)$ is at most $O\left(l^{2} \log \left(r_{*} / l\right)\right)$ for $r_{1}, r_{3} \lesssim r_{*}$. When $r_{1}, r_{3} \gtrsim r_{*}$, the argument of $\mu$ cannot be small. Then, we can use the bound $\mu(s)<l / 2 s^{2}$. Therefore, $\mathcal{O}_{r_{1}}^{[1]} \mathcal{O}_{r_{3}}^{[2]} v\left(r_{1}, r_{3}\right)$ is $O\left(l^{2}\right)$ for $r_{1}, r_{3} \gtrsim r_{*}$.

To conclude that $A_{S[K, K ; 0]}+A_{S[K, K ; K]}$ is at most $O\left(\left(l^{2} / r_{*}^{2}\right) \log \left(r_{*} / l\right)\right)$, we have to examine whether $\left[\mathcal{O}_{r_{1}}^{[1]} \mathcal{O}_{r_{3}}^{[2]} v\left(r_{1}, r_{3}\right)\right]_{r_{1}=r_{3}=r}$ behaves well at $r \rightarrow \infty$ and at $r$ $\rightarrow 0$ so that the operation of $\int d^{3} x^{\prime} R^{-2}(R+l)$ is well defined.

First we consider the large $r$ limit. Since $r_{2}$ and $r_{4}$ are bounded by the radius of the star, $\mu\left(R_{12}+R_{34}\right)$ can be replaced with $\mu\left(r_{1}+r_{3}\right) \approx l /\left(r_{1}+r_{3}\right)^{2}$ for large $r_{1}$ and/or $r_{3}$, and hence $v\left(r_{1}, r_{3}\right) \approx l^{2}\left(\kappa_{4} M_{*}\right)^{2} / r_{1} r_{3}\left(r_{1}+r_{3}\right)^{2}$. The operation of $\mathcal{O}_{r_{1}}^{[1]}$ and $\mathcal{O}_{r_{3}}^{[2]}$ does not make the fall off worse. For small $r_{1}$,

$$
\begin{aligned}
v\left(r_{1}, r_{3}\right)= & \frac{\kappa_{4}^{2} l}{r_{3}} \int_{0}^{r_{*}} d r_{2} r_{2} \Sigma^{(1)}\left(r_{2}\right) \int_{0}^{r_{*}} d r_{4} r_{4} \Sigma^{(1)}\left(r_{4}\right) \\
& \times \int_{\left|r_{3}-r_{4}\right|}^{r_{3}+r_{4}} d R_{34}\left(\mu\left(r_{2}+R_{34}\right)\right. \\
& \left.+\frac{r_{1}^{2}}{6} \mu^{\prime \prime}\left(r_{2}+R_{34}\right)+O\left(r_{1}^{4}\right)\right) \\
= & \frac{\kappa_{4}^{2} l}{r_{3}} \int_{0}^{r_{*}} d r_{2}\left[\left(1+\frac{r_{1}^{2}}{6} \partial_{r_{2}}^{2}+O\left(r_{1}^{4}\right)\right) r_{2} \Sigma^{(1)}\left(r_{2}\right)\right]
\end{aligned}
$$

$$
\times \int_{0}^{r_{*}} d r_{4} r_{4} \Sigma^{(1)}\left(r_{4}\right) \int_{\left|r_{3}-r_{4}\right|}^{r_{3}+r_{4}} d R_{34} \mu\left(r_{2}+R_{34}\right)
$$

Therefore, $\mathcal{O}_{r_{1}}^{[1]} v\left(r_{1}, r_{3}\right)$ is finite at $r_{1} \rightarrow 0$.

For $A_{S[K, 0 ; K]}$, a similar expression is obtained as

$$
\begin{aligned}
A_{S[K, 0 ; K]}= & \frac{2}{r_{*}^{2}} \int d r^{\prime} r^{\prime}\left[\mathcal{O}_{r_{3}}^{[1]} w\left(r_{1}, r_{2}, r_{3}\right)\right]_{r_{1}=r, r_{2}=r_{3}=r^{\prime}} \\
& \times \mathcal{O}_{r^{\prime}}^{[2]} \mathcal{A}_{0}^{(1)}\left(r^{\prime}\right),
\end{aligned}
$$

with

$$
\begin{aligned}
w\left(r_{1}, r_{2}, r_{3}\right) \equiv & r_{2} \int d \Omega_{2} \int_{0}^{\infty} d y^{\prime} G_{K}\left(\mathbf{x}_{1}, 0 ; \mathbf{x}_{2}, y^{\prime}\right) \mathcal{A}_{K}^{(1)}\left(r_{3}, y^{\prime}\right) \\
= & \frac{\kappa_{4} l}{4 r_{1} r_{3}} \int_{0}^{r_{*}} d r_{4} r_{4} \Sigma^{(1)}\left(r_{4}\right) \int_{\left|r_{1}-r_{2}\right|}^{r_{1}+r_{2}} d R_{12} \\
& \times \int_{\left|r_{3}-r_{4}\right|}^{r_{3}+r_{4}} d R_{34} \mu\left(R_{12}+R_{34}\right),
\end{aligned}
$$

and $\mathcal{A}_{0}^{(1)}$ represents the part of $\mathcal{A}^{(1)}$ propagated by $G_{0}$. In the same way, we can show that $A_{S[K, 0 ; K]}$ is at most $O\left(\left(l^{2} / r_{*}^{2}\right) \log \left(r_{*} / l\right)\right)$.

\section{2. $A_{Q[K, K ; 0]}$ and $A_{Q[K, K ; K]}$}

$A_{Q[K, K ; 0]}$ and $A_{Q[K, K ; K]}$ are also bounded as

$$
\begin{aligned}
\left|A_{Q[K, K ; 0]}+A_{Q[K, K ; K]}\right| & \\
< & \frac{1}{2 \pi l} \int d^{3} x^{\prime} \frac{R+l}{R^{2}} \int_{0}^{\infty} d y a^{4}(y) Q_{y y}(\mathbf{x}, y) \\
\leqslant & \frac{1}{8 \pi l} \int d^{3} x^{\prime} \frac{R+l}{R^{2}}\left\{\mid \mathcal{A}_{, y}^{(1)} \mathcal{A}_{K}^{(1)}+\mathcal{B}_{, y}^{(1)} \mathcal{B}_{K}^{(1)}\right. \\
& +\left.2 \mathcal{C}_{, y}^{(1)} \mathcal{C}_{K}^{(1)}\right|_{y=0}+\mid \int_{0}^{\infty} d y\left(\left(a^{4} \mathcal{A}_{, y}^{(1)}\right)_{, y} \mathcal{A}_{K}^{(1)}\right. \\
& \left.\left.+\left(a^{4} \mathcal{B}_{, y}^{(1)}\right)_{, y} \mathcal{B}_{K}^{(1)}+2\left(a^{4} \mathcal{C}_{, y}^{(1)}\right)_{, y} \mathcal{C}_{K}^{(1)}\right) \mid\right\} .
\end{aligned}
$$

The first term in the curly brackets is at most $O\left(\left(l^{3} / r_{*}^{4}\right) \log \left(r_{*} / l\right)\right)$. By using the relations obtained from Eq. (2.32),

$$
\begin{aligned}
& \frac{1}{a^{4}}\left(a^{4} \mathcal{A}_{, y}^{(1)}\right)_{, y}=-a^{-2} \triangle \mathcal{A}^{(1)}+2 \kappa_{5} \Sigma^{(1)} \delta(y), \\
& \frac{1}{a^{4}}\left(a^{4} \mathcal{B}_{, y}^{(1)}\right)_{, y}=a^{-2} \frac{1}{r} \partial_{r} \mathcal{A}^{(1)}-\frac{2 \kappa_{5}}{r^{3}} \int d r r^{2} \Sigma^{(1)} \delta(y),
\end{aligned}
$$


and $\mathcal{C}^{(1)}=-\left(\mathcal{A}^{(1)}+\mathcal{B}^{(1)}\right) / 2$, the second term is reduced to the form in Eq. (4.16). Therefore, the contribution to $A_{Q}$ from this term is at most $O\left(\left(l^{2} / r_{*}^{2}\right) \log \left(r_{*} / l\right)\right)$ for the same reason.

\section{SUMMARY}

In this paper we developed second order perturbations in the RS single brane model, restricting the configuration to the static axisymmetric one. From the five-dimensional Einstein equations, we derived the master equations for second order perturbations. At the level of linear perturbations, we can use the RS gauge, in which all perturbation equations are decoupled. Since the transverse-traceless condition cannot be imposed on the second order, the second order perturbative equations are inevitably coupled. As we have shown, however, the four-dimensional spatial components of the second order metric function $\mathcal{B}^{(2)}$ and $\mathcal{C}^{(2)}$ turned out to be concisely represented by the temporal component $\mathcal{A}^{(2)}$ with the first order metric functions. Therefore, the problem was reduced to solving a single differential equation for $\mathcal{A}^{(2)}$. Once we solve for $\mathcal{A}^{(2)}$, the other metric functions $\mathcal{B}^{(2)}$ and $\mathcal{C}^{(2)}$ follow from it. Further, to discuss the metric induced on the three-brane, we introduced the GN gauge in which a hypersurface with constant fifth-coordinate coincides with the location of the three-brane. we gave the second order gauge transformations between the RS gauge and the GN gauge explicitly.

Based on this formulation, we first discussed the zero mode truncation for second order perturbations. It was shown that the metric induced on the three-brane evaluated by using the approximation of the zero mode truncation exactly agrees with that for the four-dimensional Einstein gravity.

Next, we evaluated the contribution to the metric functions from the KK modes. Since the mode-by-mode analysis shows a pathological feature even at the level of linear perturbations, it is necessary to sum up all the mass eigenvalues to handle the KK mode interactions. Performing such an analysis, we have confirmed that the correction due to the KK mode coupling on the induced metric is suppressed by a factor of $O\left(\left(l^{2} / r_{*}^{2}\right) \log \left(r_{*} / l\right)\right)$, and there appears no pathological behavior. We therefore conclude that second order perturbations in the RS single brane model behave well and the result basically agrees with the prediction by fourdimensional Einstein gravity. The relative order of the correction is $O\left(\left(l^{2} / r_{*}^{2}\right) \log \left(r_{*} / l\right)\right)$. In the language of the postNewtonian (PN) analysis of the four-dimensional Einstein gravity, the order of $\mathcal{A}^{(2)}$ and $\mathcal{B}^{(1)}$ is $1 \mathrm{PN}$, and that of $\mathcal{B}^{(2)}$ is $2 \mathrm{PN}$. According to the parametrized post-Newtonian (PPN) formalism, the PPN parameters at the 1PN order, $\beta$ and $\gamma$, are observationally constrained to the accuracy of about $0.1 \%$ or so [32]. Although this accuracy might be improved in the future, there will be no chance, unfortunately, to find the evidence for the large extra dimension from the precision test of the solar system since the predicted deviation in $\beta, \gamma$ is $O\left(\left(l^{2} / r_{*}^{2}\right) \log \left(r_{*} / l\right)\right)$.

Of course, since our discussion developed in the present paper is restricted to second order perturbations with spheri- cal symmetry, the gravity induced on the three-brane in the RS single brane model might deviate from the fourdimensional Einstein gravity in a more general situation. Hence, it would be necessary to develop a more complete proof of the coincidence including all higher order terms. Although we did not discuss the RS two brane model, it is also interesting to study the post-Newtonian correction in this model. The analysis is now in progress and will be reported soon in a separate paper.

\section{ACKNOWLEDGMENTS}

H.K. would like to thank Takashi Nakamura and Hideo Kodama for informative comments and discussion. We would also like to thank V. F. Mukhanov for informing us about Ref. [34]. This work is supported by the Monbukagakusho Grant-in-Aid No. 1270154.

\section{APPENDIX A: DERIVATION OF $\overline{\mathcal{B}}^{(2)}$}

As was mentioned in the text below Eq. (4.4), the expression given in the last line of Eq. (4.4) contains the terms with $y$ integration. They are explicitly written as

$$
\triangle^{-1}\left(-\frac{3}{l} \int_{0}^{\infty} d y a^{2} Q_{y y}+\frac{1}{r^{2}} \partial_{r} \int_{0}^{\infty} d y r^{2} Q_{y r}\right) .
$$

(2)

The first integral comes from $\hat{\xi}^{y}$ contained in $\delta \mathcal{A}^{(2)}, \delta \mathcal{B}^{(2)}$, and $\delta \mathcal{C}^{(2)}$. In obtaining the expression (4.5) from (4.4), how to reduce these terms will be the only nontrivial manipulation. The rest of the computation is slightly complicated but almost straightforward.

To rewrite the first integral, we use the relation

$$
\begin{aligned}
-\frac{1}{2} Q_{y y}(r, 0)= & \frac{1}{8} \int_{0}^{\infty} d y \partial_{y}\left\{a ^ { - 6 } \left[\left(a^{4} \mathcal{A}_{, y}^{(1)}\right)^{2}+\left(a^{4} \mathcal{B}_{, y}^{(1)}\right)^{2}\right.\right. \\
& \left.\left.+2\left(a^{4} \mathcal{C}_{, y}^{(1)}\right)^{2}\right]\right\} \\
= & \frac{3}{l} \int_{0}^{\infty} d y a^{2} Q_{y y}+\frac{1}{8} \int_{0}^{\infty} d y \frac{1}{a^{2}}\left[\left(3 \mathcal{A}_{, y}^{(1)}+\mathcal{B}_{, y}^{(1)}\right)\right. \\
& \left.\times\left(a^{4} \mathcal{A}_{, y}^{(1)}\right)_{, y}+\left(3 \mathcal{B}_{, y}^{(1)}+\mathcal{A}_{, y}^{(1)}\right)\left(a^{4} \mathcal{B}_{, y}^{(1)}\right)_{, y}\right],
\end{aligned}
$$

where we have used the traceless condition $\mathcal{C}^{(1)}=-\left(\mathcal{A}^{(1)}\right.$ $\left.+\mathcal{B}^{(1)}\right) / 2$. On the other hand, the second part is rewritten as

$$
\begin{aligned}
\frac{1}{r^{2}} \partial_{r} \int_{0}^{\infty} d y r^{2} Q_{y r}= & -\frac{1}{2} Q_{r r}+\frac{1}{8} \int_{0}^{\infty} d y\left[\left(3 \mathcal{A}_{, y}^{(1)}+\mathcal{B}_{, y}^{(1)}\right) \triangle \mathcal{A}^{(1)}\right. \\
& \left.+\left(3 \mathcal{B}_{, y}^{(1)}+\mathcal{A}_{, y}^{(1)}\right) \triangle \mathcal{B}^{(1)}\right],
\end{aligned}
$$

where again we have used the traceless condition. After the substitution of these relations and Eq. (4.24), the remaining $y$ integration is just

$$
\frac{1}{8} \int_{0}^{\infty} d y\left(3 \mathcal{B}_{, y}^{(1)}+\mathcal{A}_{, y}^{(1)}\right)\left[\triangle+\frac{1}{a^{2}} \partial_{y} a^{4} \partial_{y}\right] \mathcal{B}^{(1)} .
$$


We can derive

$$
\frac{1}{a^{2}}\left(a^{4} \mathcal{B}_{, y}^{(1)}\right)_{, y}=-\left(\Delta \mathcal{B}^{(1)}+\frac{2}{r} \mathcal{B}_{, r}^{(1)}\right)-\frac{2 \kappa_{5}}{r^{3}} \int d r r^{2} \Sigma^{(1)} \delta(y)
$$

from Eq. (4.25), and $\left(3 \mathcal{B}_{, y}^{(1)}+\mathcal{A}_{, y}^{(1)}\right)=-r \mathcal{B}_{, r y}^{(1)}$ from Eq. (2.18). With the aid of these relations, it is easy to see that the remaining $y$ integration (A2) can be performed.

\section{APPENDIX B: SECOND ORDER PERTURBATIONS IN THE 4D EINSTEIN GRAVITY}

In this appendix, we give second order perturbations in the four-dimensional Einstein gravity for the comparison with the zero mode truncation of the gravity in the RS single brane model. Although the results are well known as the second post-Newtonian analysis [33], we present a brief derivation for the following two reasons. (1) Since we are working in a specific gauge, we need to consider a gauge transformation to compare our results with the expression presented in a different gauge. (2) To compute metric perturbations in our restricted situation from the beginning is much easier than to follow a literature in which unrestricted cases are discussed.

We assume that the four-dimensional metric is static and isotropic,

$$
d s^{2}=-e^{A(r)} d t^{2}+e^{B(r)} d \mathbf{x}^{2} .
$$

Up to the second order, the four-dimensional Einstein equations with the energy-momentum tensor given in Eq. (2.2) are

$$
\begin{aligned}
R_{t}^{t} & =\frac{1}{2}(B-1) \triangle A-\frac{1}{4} A_{, r}(A+B)_{, r}=-\frac{\kappa_{4}}{2}(\rho+3 P), \\
R_{r}^{r} & =\frac{1}{2}(B-1)\left(\triangle(A+2 B)-\frac{2}{r}(A+B)_{, r}\right)+\frac{1}{4} A_{, r}(B-A)_{, r} \\
& =\frac{\kappa_{4}}{2}(\rho-P), \\
R_{\theta}^{\theta} & =\frac{1}{2}(B-1)\left(\triangle B+\frac{1}{r}(A+B)_{, r}\right)-\frac{1}{4} B_{, r}(A+B)_{, r} \\
& =\frac{\kappa_{4}}{2}(\rho-P) .
\end{aligned}
$$

We expand $A$ and $B$ to the second order as Eq. (2.9). Solving these equations at the linear order, we obtain $A^{(1)}=-B^{(1)}$ $=2 \phi$, where $\phi(r)$ is similarly defined as in Eq. (3.10). Putting these results into Eq. (B1), the equation for $A^{(2)}$ becomes

$$
\triangle A^{(2)}=\kappa_{4}\left(\rho^{(2)}+3 P\right)-4 \phi \triangle \phi,
$$

which agrees with Eq. (4.8). Eliminating $\triangle A^{(2)}$ and $P$ from Eqs. (B1), (B2), and (B3), we obtain

$$
\triangle B^{(2)}=-\kappa_{4} \rho^{(2)}+4 \phi \triangle \phi-\left(\phi_{, r}\right)^{2} .
$$

Hence it was shown that the result obtained by the zero mode truncation (4.9) agrees with that for the four-dimensional Einstein gravity (B5).
[1] N. Arkani-Hamed, S. Dimopoulos, and G. Dvali, Phys. Lett. B 429, 263 (1998); Phys. Rev. D 59, 086004 (1999).

[2] I. Antoniadis, N. Arkani-Hamed, S. Dimopoulos, and G. Dvali, Phys. Lett. B 436, 257 (1998).

[3] L. Randall and R. Sundrum, Phys. Rev. Lett. 83, 3370 (1999).

[4] L. Randall and R. Sundrum, Phys. Rev. Lett. 83, 4690 (1999).

[5] J. Garriga and T. Tanaka, Phys. Rev. Lett. 84, 2778 (2000).

[6] T. Shiromizu, K. Maeda, and M. Sasaki, Phys. Rev. D 62, 024012 (2000).

[7] M. Sasaki, T. Shiromizu, and K. Maeda, Phys. Rev. D 62, 024008 (2000).

[8] U. Gen and M. Sasaki, gr-qc/0011078.

[9] T. Tanaka and X. Montes, Nucl. Phys. B582, 259 (2000).

[10] S.B. Giddings, E. Katz, and L. Randall, J. High Energy Phys. 03, 023 (2000).

[11] T. Tanaka, Prog. Theor. Phys. 104, 545 (2000).

[12] C. Csaki, M. Graesser, L. Randall, and J. Terning, Phys. Rev. D 62, 045015 (2000).

[13] C. Charmousis, R. Gregory, and V.A. Rubakov, Phys. Rev. D 62, 067505 (2000).

[14] T. Chiba, Phys. Rev. D 62, 021502(R) (2000).

[15] W.D. Goldberger and M.B. Wise, Phys. Rev. Lett. 83, 4922 (1999).
[16] W.D. Goldberger and M.B. Wise, Phys. Lett. B 475, 275 (2000).

[17] O. DeWolfe, D.Z. Freedman, S.S. Gubser, and A. Karch, Phys. Rev. D 62, 046008 (2000).

[18] H. Kodama, A. Ishibashi, and O. Seto, Phys. Rev. D 62, 064022 (2000).

[19] S. Mukohyama, Phys. Rev. D 62, 084015 (2000); Class. Quantum Grav. 17, 4777 (2000).

[20] R. Maartens, Phys. Rev. D 62, 084023 (2000).

[21] K. Koyama and J. Soda, Phys. Rev. D 62, 123502 (2000).

[22] C. van de Bruck, M. Dorca, R.H. Brandenberger, and A. Lukas, Phys. Rev. D 62, 123515 (2000).

[23] D. Langlois, Phys. Rev. D 62, 126012 (2000).

[24] A. Chamblin and G.W. Gibbons, Phys. Rev. Lett. 84, 1090 (2000).

[25] A. Chamblin, S.W. Hawking, and H.S. Reall, Phys. Rev. D 61, 065007 (2000).

[26] R. Gregory and R. Laflamme, Phys. Rev. Lett. 70, 2837 (1993).

[27] R. Gregory, Class. Quantum Grav. 17, L125 (2000).

[28] A. Chamblin, H.S. Reall, H. Shinkai, and T. Shiromizu, Phys. Rev. D 63, 064015 (2001).

[29] R. Emparan, G.T. Horowitz, and R.C. Myers, J. High Energy Phys. 01, 007 (2000). 
[30] N. Dadhich, R. Maartens, P. Papadopoulos, and V. Rezania, Phys. Lett. B 487, 1 (2000).

[31] W. Israel, Nuovo Cimento B 44, 1 (1966).

[32] C.M. Will, Theory and Experiment in Gravitational Physics, revised ed. (Cambridge University Press, Cambridge, England,
1993); lecture notes from the 1998 SLAC Summer Institute on Particle Physics, gr-qc/9811036.

[33] For example, S. Chandrasekhar and Y. Nutku, Astrophys. J. 158, 55 (1969); L. Blanchet, Phys. Rev. D 51, 2559 (1995). [34] I. Giannakis and H. Ren, Phys. Rev. D 63, 024001 (2001). 